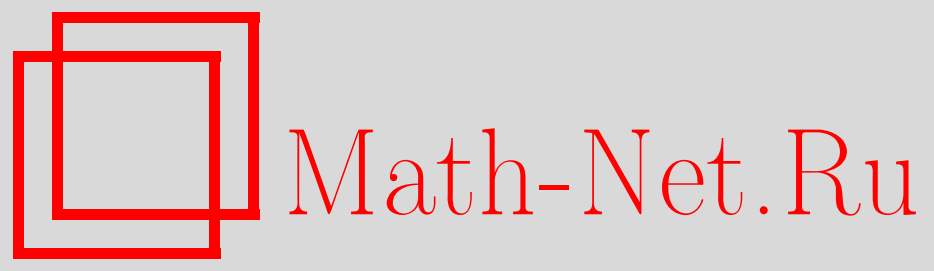

Р. А. Зубов, С. А. Пастон, Е. В. Прохватилов, Точное решение уравнения 'т Хоофта в пределе тяжелых кварков разной массы, ТМФ, 2015, том 184, номер 3, 449-455

DOI: https://doi.org/10.4213/tmf8925

Использование Общероссийского математического портала Math-Net.Ru подразумевает, что вы прочитали и согласны с пользовательским соглашением http://www.mathnet.ru/rus/agreement

Параметры загрузки:

IP: 54.166 .219 .16

26 апреля 2023 г., 16:05:30

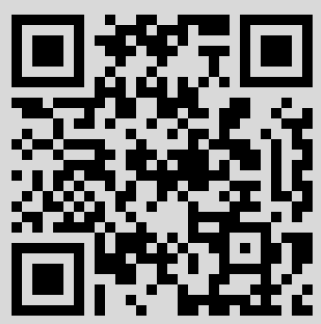


Том 184, № 3

сентябрь, 2015

(C) 2015 г. $\quad$ Р. А. Зубов*, С. А. Пастон*, Е. В. Прохватилов*

\section{ТОЧНОЕ РЕШЕНИЕ УРАВНЕНИЯ 'Т ХООФТА В ПРЕДЕЛЕ ТЯЖЕЛЫХ КВАРКОВ РАЗНОЙ МАССЫ}

Рассматривается уравнение 'т Хоофта для связанных состояний в двумерной квантовой хромодинамике в пределе бесконечного числа цветов. Для случая кварков разной массы в пределе стремления их масс к бесконечности получено приближение для низкоэнергетической части спектра и соответствующих волновых функций. Показано, что, как и в частном случае равных масс, уравнение 'т Хоофта сводится в первом приближении к уравнению Шредингера с линейным потенциалом, т. е. к уравнению для частицы в "треугольной" потенциальной яме. Также обсуждается возможность получения поправок к этому приближению.

Ключевые слова: уравнение 'т Хоофта, двумерная квантовая хромодинамика, световой фронт.

DOI: $10.4213 / \operatorname{tmf} 8925$

\section{1. ВВЕДЕНИЕ}

В 1974 году 'т Хоофт исследовал модель КХД с локальной калибровочной группой $S U(N)$ в двух измерениях [1]. Она задается лагранжианом

$$
\mathcal{L}=-\frac{1}{2} \operatorname{tr}\left(G_{\rho \nu} G^{\rho \nu}\right)+\sum_{a} \bar{q}^{a}\left(i \gamma^{\rho} D_{\rho}-m_{a}\right) q^{a} .
$$

Здесь тензор $G_{\rho \nu}=(i / g)\left[D_{\rho}, D_{\nu}\right]$ и ковариантная производная $D_{\rho}=\partial_{\rho}-i g A_{\rho}$ заданы через потенциал глюонного поля $A_{\rho}$, представленный бесследовыми эрмитовыми $(N \times N)$-матрицами, а $q^{a}$ - это описывающие кварки фермионные поля с массами $m_{a}$. В пределе $N \rightarrow \infty$ при фиксированном $g^{2} N$ 'т Хоофту удалось показать, что в этой модели присутствуют только связанные двухкварковые мезонные состояния. Их спектр точно описывается вытекающим из уравнения Бете-Солпитера в светоподобной калибровке $A_{-}=A^{+}=0$ интегральным уравнением

$$
\mu^{2} \varphi(\xi)=\left(\frac{\alpha_{1}}{\xi}+\frac{\alpha_{2}}{1-\xi}\right) \varphi(\xi)-\mathrm{P} \int_{0}^{1} \frac{\varphi\left(\xi^{\prime}\right)}{\left(\xi^{\prime}-\xi\right)^{2}} d \xi^{\prime}
$$

* Санкт-Петербургский государственный университет, Санкт-Петербург, Россия. E-mail: roman.zubov@hep.phys.spbu.ru, paston@pobox.spbu.ru, evgeni.prokhvat@pobox.spbu.ru 
Здесь $0 \leqslant \xi \leqslant 1$ - это относительный светоподобный импульс одного из кварков, $\alpha_{1,2}$ связаны с голыми массами кварков $m_{1,2}$ из лагранжиана,

$$
\alpha_{1,2}=\frac{\pi}{g^{2}} m_{1,2}^{2}-1,
$$

$g$ - константа связи, имеющая в двух измерениях размерность массы, а $\mu$ - масса связанного состояния в единицах $g / \sqrt{\pi}$. В уравнении (2) интеграл понимается в смысле главного значения:

$$
\mathrm{P} \int \frac{\varphi(k)}{k^{2}} d k=\frac{1}{2} \int \frac{\varphi(k+i \varepsilon)}{(k+i \varepsilon)^{2}} d k+\frac{1}{2} \int \frac{\varphi(k-i \varepsilon)}{(k-i \varepsilon)^{2}} d k .
$$

Заметим, что уравнение 'т Хоофта (2) фактически соответствует квантованию теории на световом фронте [2], [3]. Вопросы, связанные с эквивалентностью такого квантования и обычного квантования в лоренцевых координатах, обсуждаются в работах [4]-[7].

Уравнение, подобное уравнению 'т Хоофта (2), встречается и в других двумерных теориях, в которых есть потенциал конфайнмента. Примером является полевая теория Изинга (Ising Field Theory) [8], [9]. В отличие от модели 'т Хоофта, в этой теории аналогичное уравнение описывает некоторое приближение к истинному спектру, однако оно проливает свет на спектр полной теории. Также аналог уравнения 'т Хоофта и связанные с ним идеи используются в некоторых феноменологических моделях и голографии [10]-[12]. В работах [13], [14] рассматриваемая 'т Хоофтом двумерная модель применяется к проблеме нарушения кварк-адронной дуальности. В свою очередь в статьях [15], [16] отмечается связь некоторых вопросов теории волн на поверхности жидкости с частным случаем $\alpha_{1,2}=0$ уравнения (2).

Уравнение 'т Хоофта может быть решено численно с любой степенью точности, и к нынешнему моменту существуют различные способы это сделать [1], [9], [17]-[20]. Тем не менее из-за важности и простоты этого уравнения имеется интерес и к его аналитическому решению [15], [21]--[26], в частности к возможности рассматривать параметры уравнения $\alpha_{1,2}$ в комплексной области. В работе [26] рассматривается специальный случай $\alpha_{1}=\alpha_{2}=0$, а также приводятся предварительные результаты для более общего случая $\alpha_{1}=\alpha_{2}=\alpha$. В работе [25] также рассматривается случай равных масс и исследуются асимптотические свойства уравнения 'т Хоофта в пределе $\alpha \rightarrow \infty$, т. е. случай больших масс кварков. В зависимости от рассматриваемой области спектра получаются разные асимптотики: низкоэнергетическое (для низших возбуждений в спектре) и полуклассическое (для больших собственных значений) разложения. Кроме того, в рамках полуфеноменологического приближения из работы [27] можно получить уравнение, обобщающее двумерное уравнение 'т Хоофта для моделей реальной КХД в $3+1$ и $2+1$ измерениях. Этому вопросу авторы планируют посвятить отдельную работу.

В настоящей работе мы рассматриваем низкоэнергетическую часть спектра в общем случае $\alpha_{1} \neq \alpha_{2}$ в пределе больших масс кварков (при $\left.\alpha_{1,2} \rightarrow \infty\right)$. Мы используем метод, основанный на решении уравнения в фурье-сопряженных координатах [8] (см. также приложение А в работе [25]). Полученный результат показывает, что, как и в частном случае равных масс, уравнение 'т Хоофта сводится в первом приближении к уравнению Шредингера с линейным потенциалом $|x|$, т. е. к уравнению для частицы в “треугольной" потенциальной яме. Дальнейшие поправки к этому решению можно получить, развивая теорию возмущений. 


\section{2. ПРЕДЕЛ ТЯЖЕЛЫХ КВАРКОВ}

Начнем с оригинального уравнения 'т Хоофта (2). Поскольку мы хотим рассматривать предел больших масс кварков, введем следующую параметризацию:

$$
\alpha_{1} \equiv a_{1}^{2} \alpha, \quad \alpha_{2} \equiv a_{2}^{2} \alpha,
$$

где $\alpha$ будет стремиться к бесконечности, а оба параметра $a_{1}$ и $a_{2}$ имеют порядок $O(1)$. Заметим, что $a_{1} \sqrt{\alpha}$ и $a_{2} \sqrt{\alpha}$ имеют смысл обезразмеренных масс кварков в указанном пределе, поскольку $\alpha_{1,2} \approx\left(\pi / g^{2}\right) m_{1,2}^{2}$. Теперь перейдем к новой переменной

$$
\xi=\omega+k_{1},
$$

где

$$
k_{1} \equiv \frac{a_{1}}{a_{1}+a_{2}}, \quad k_{2} \equiv 1-k_{1}=\frac{a_{2}}{a_{1}+a_{2}} .
$$

В результате уравнение (2) примет вид

$$
\mu^{2} \varphi(\omega)=\left(\frac{a_{1}^{2}}{k_{1}+\omega}+\frac{a_{2}^{2}}{k_{2}-\omega}\right) \alpha \varphi(\omega)-\mathrm{P} \int_{-k_{1}}^{k_{2}} \frac{\varphi\left(\omega^{\prime}\right)}{\left(\omega^{\prime}-\omega\right)^{2}} d \omega^{\prime} .
$$

Рассмотрим это уравнение в пределе $\alpha \rightarrow \infty$. В главном порядке низшее значение $\mu_{(0)}^{2}$ в спектре соответствует минимальному значению первого слагаемого в правой части уравнения, этот минимум при выбранном сдвиге $(6),(7)$ достигается при $\omega=0$, поэтому

$$
\mu_{(0)}^{2}=\left(a_{1}+a_{2}\right)^{2} \alpha .
$$

При этом соответствующая собственная функция стремится к дельта-функции Дирака $\delta(\omega)$ при $\alpha \rightarrow \infty$.

Будем искать поправку к значению $\mu^{2}$ в виде

$$
\mu^{2}=\mu_{(0)}^{2}+\gamma
$$

В результате соответствующей подстановки уравнение приобретает вид

$$
\gamma \varphi(\omega)=-\frac{\left(a_{1}+a_{2}\right)^{2} \omega^{2} \alpha}{\left(k_{1}+k_{2}\right)^{2} \omega^{2}+\left(k_{1}^{2}-k_{2}^{2}\right) \omega-k_{1} k_{2}} \varphi(\omega)-\mathrm{P} \int_{-k_{1}}^{k_{2}} \frac{\varphi\left(\omega^{\prime}\right)}{\left(\omega^{\prime}-\omega\right)^{2}} d \omega^{\prime} .
$$

Далее введем обозначения

$$
t \equiv \alpha^{-1 / 3}, \quad \bar{\gamma} \equiv \gamma t
$$

и сделаем "растяжение" независимой переменной

$$
\omega=s t, \quad \varphi(\omega)=\varphi(s t)=f(s),
$$

получим уравнение

$$
\bar{\gamma} f(s)=-\frac{\left(a_{1}+a_{2}\right)^{2} s^{2}}{\left(k_{1}+k_{2}\right)^{2} s^{2} t^{2}+\left(k_{1}^{2}-k_{2}^{2}\right) s t-k_{1} k_{2}} f(s)-\mathrm{P} \int_{-k_{1} / t}^{k_{2} / t} \frac{f\left(s^{\prime}\right)}{\left(s^{\prime}-s\right)^{2}} d s^{\prime} .
$$


В этом уравнении можно сделать формальный переход к пределу $t \rightarrow 0$, соответствующий пределу $\alpha \rightarrow \infty$. После этого получится уравнение, уже не содержащее $t$ и, таким образом, соответствующее предельному случаю в первом приближении. Это уравнение имеет вид

$$
\bar{\gamma} f(s)=\frac{\left(a_{1}+a_{2}\right)^{4}}{a_{1} a_{2}} s^{2} f(s)-\mathrm{P} \int_{-\infty}^{\infty} \frac{f\left(s^{\prime}\right)}{\left(s^{\prime}-s\right)^{2}} d s^{\prime} .
$$

Следует отметить, что в настоящий момент не существует строгого доказательства корректности проделанного формального предельного перехода, этот вопрос требует дополнительного исследования. Если величину расхождения, возникающего при взятии предела в первом слагаемом правой части уравнения (14), несложно оценить путем разложения в ряд, то для соответствующего расхождения, связанного с заменой конечных пределов интегрирования бесконечными во втором слагаемом, ее оценить гораздо труднее. В работе [25] это последнее расхождение оценивается как величина, много меньшая $t^{4}$, но является ли эта оценка достаточно строго обоснованной - неясно.

Теперь в уравнении (15) легко сделать преобразование Фурье

$$
\hat{f}(x)=\frac{1}{2 \pi} \int_{-\infty}^{\infty} f(s) e^{i s x} d s
$$

и получить уравнение в “координатной” области

$$
\bar{\gamma} \hat{f}(x)=-\frac{\left(a_{1}+a_{2}\right)^{4}}{a_{1} a_{2}} \hat{f}^{\prime \prime}(x)+\pi|x| \hat{f}(x) .
$$

Заметим, что если $\alpha_{1}=\alpha_{2}$ (т. е. $k_{1}=k_{2}=1 / 2$ ), то $x$ пропорционально расстоянию между кварком и антикварком в $x^{-}$-направлении.

Полученное уравнение есть не что иное, как уравнение Шредингера для частицы в “треугольной" потенциальной яме, т. е. с потенциалом вида $a|x|$. Оно имеет следующее решение:

$$
\begin{aligned}
\bar{\gamma}_{n} & =-\left(\pi^{2} \frac{\left(a_{1}+a_{2}\right)^{4}}{a_{1} a_{2}}\right)^{1 / 3} z_{n}, \quad n=0,1, \ldots, \\
\hat{f}_{n}(x) & = \begin{cases}\operatorname{Ai}\left[\left(\pi \frac{a_{1} a_{2}}{\left(a_{1}+a_{2}\right)^{4}}\right)^{1 / 3} x+z_{n}\right], & \text { если } x \geqslant 0, \\
(-1)^{n} \hat{f}_{n}(-x), & \text { если } \quad x<0 .\end{cases}
\end{aligned}
$$

Здесь через $z_{2 k+1}$ обозначены нули функции Эйри, а через $z_{2 k}-$ нули ее производной $(k=0,1, \ldots)$. Существует асимптотика величин $z_{n}$ для больших $n[28]$ :

$$
z_{n} \approx-\left[\frac{3}{4} \pi\left(n+\frac{1}{2}\right)\right]^{2 / 3}
$$


Эта формула обладает точностью $1 \%$ уже для $n=1$ и гораздо лучшей для больших $n$. Таким образом, можно написать

$$
\bar{\gamma}_{n} \approx\left[\left(\frac{3}{4} \pi^{2}\right)^{2} \frac{\left(a_{1}+a_{2}\right)^{4}}{a_{1} a_{2}}\right]^{1 / 3}\left(n+\frac{1}{2}\right)^{2 / 3}, \quad n=0,1, \ldots
$$

Теперь, возвращаясь к исходным переменным, можно записать выражения для масс $\mu_{n}$ связанных состояний и соответствующих им функций $\varphi_{n}(\omega)$, являющихся при большом $\alpha$ в первом приближении решением уравнения 'т Хоофта (2):

$$
\begin{gathered}
\mu_{n}^{2}=-\left(\pi^{2} \alpha \frac{\left(a_{1}+a_{2}\right)^{4}}{a_{1} a_{2}}\right)^{1 / 3} z_{n}+\left(a_{1}+a_{2}\right)^{2} \alpha \approx \\
\approx\left[\left(\frac{3}{4} \pi^{2}\right)^{2} \frac{\left(a_{1}+a_{2}\right)^{4}}{a_{1} a_{2}} \alpha\right]^{1 / 3}\left(n+\frac{1}{2}\right)^{2 / 3}+\left(a_{1}+a_{2}\right)^{2} \alpha \\
\varphi_{n}(\omega)=\int_{-\infty}^{\infty} \hat{f}_{n}(x t) e^{-i \omega x} d x
\end{gathered}
$$

где $n=0,1, \ldots$.

Мы получили решение уравнения 'т Хоофта с неравными массами кварков в первом приближении в пределе больших масс. Чтобы найти поправки к этому решению, нужно разложить все члены в уравнении (14) по степеням $t$ и решить полученное уравнение по теории возмущений. В работе [25] высказано утверждение о том, что при разложении интеграла в (14) в ряд по $t$ поправки к главному значению много меньше, чем $t^{4}$. Таким образом, если ограничиться порядком точности $O\left(t^{4}\right)$, то в этом интеграле можно заменить пределы $\left(-k_{1} / t, k_{2} / t\right)$ на $(-\infty, \infty)$. Далее для краткости мы выписываем члены вплоть до порядка $O\left(t^{2}\right)$ :

$$
\begin{aligned}
\bar{\gamma} f(s)= & {\left[\frac{\left(a_{1}+a_{2}\right)^{4}}{a_{1} a_{2}} s^{2}+\frac{\left(a_{1}+a_{2}\right)^{5}\left(a_{1}-a_{2}\right)}{a_{1}^{2} a_{2}^{2}} t s^{3}+\right.} \\
& \left.+\frac{\left(a_{1}+a_{2}\right)^{6}\left(a_{1}^{2}-a_{1} a_{2}+a_{2}^{2}\right)}{a_{1}^{3} a_{2}^{3}} t^{2} s^{4}+\cdots\right] f(s)-\mathrm{P} \int_{-\infty}^{\infty} \frac{f\left(s^{\prime}\right)}{\left(s^{\prime}-s\right)^{2}} d s^{\prime} .
\end{aligned}
$$

Совершая преобразование Фурье (16), можно получить уравнение в "координатном" пространстве:

$$
\begin{aligned}
\bar{\gamma} f(s)= & {\left[|x|-\frac{\left(a_{1}+a_{2}\right)^{4}}{a_{1} a_{2}} \frac{d^{2}}{d x^{2}}+i \frac{\left(a_{1}+a_{2}\right)^{5}\left(a_{1}-a_{2}\right)}{a_{1}^{2} a_{2}^{2}} t \frac{d^{3}}{d x^{3}}+\right.} \\
& \left.+\frac{\left(a_{1}+a_{2}\right)^{6}\left(a_{1}^{2}-a_{1} a_{2}+a_{2}^{2}\right)}{a_{1}^{3} a_{2}^{3}} t^{2} \frac{d^{4}}{d x^{4}}+\cdots\right] f(s) .
\end{aligned}
$$

Оно решается по теории возмущений способом, в целом аналогичным использованному в работах [8], [25]. Однако, как мы уже отмечали, при таком подходе существенно использование оценки $O\left(t^{4}\right)$, предложенной в работе [25], правильность которой пока кажется недостаточно обоснованной. Даже если эта оценка верна, при ее использовании нельзя получить данным способом поправки порядка $O\left(t^{5}\right)$ и выше. 


\section{3. ЗАКЛЮЧЕНИЕ}

В предложенной работе получено решение уравнения 'т Хоофта в пределе больших и при этом неравных масс кварков. Путем перехода к пределу в исследуемом интегральном уравнении показано, что спектр и набор волновых функций рассматриваемой модели совпадают (после некоторых "перерастяжений") со спектром и волновыми функциями уравнения Шредингера с линейным потенциалом, т. е. уравнения для частицы в “треугольной” потенциальной яме. Полученные результаты хорошо согласуются с результатами проведенных численных расчетов, что косвенно указывает на правильность использованного предельного перехода. Однако прямое доказательство математической корректности этого предельного перехода мы не проводим. Этому вопросу планируется посвятить отдельную работу. В частности, на этом пути можно будет проверить высказанное в работе [25] утверждение об оценке скорости расхождения, появляющегося в пределе больших масс кварков при переходе от конечных пределов интегрирования к бесконечным в интеграле, входящем в уравнение 'т Хоофта.

Благодарности. Работа Р. А. Зубова и Е. В. Прохватилова выполнена при поддержке СПбГУ (грант 11.38.189.2014).

\section{Список литературы}

[1] G. 't Hooft, Nucl. Phys. B, 75:3 (1974), 461-470.

[2] A. Bassetto, L. Griguolo, F. Vian, Nucl. Phys. B, 559:1-2 (1999), 563-590, arXiv: hep-th/9906125.

[3] A. Bassetto, L. Griguolo, F. Vian, Light-front vacuum and instantons in two-dimensions, arXiv: hep-th/0004026..

[4] В. А. Франке, С. А. Пастон, ТМФ, 112:3, 399-416, arXiv: hep-th/9901110.

[5] С. А. Пастон, Е. В. Прохватилов, В. А. Франке, ТМФ, 120:3 (1999), 417-437.

[6] С. А. Пастон, Е. В. Прохватилов, В. А. Франке, ТМФ, 131:1 (2002), 84-97.

[7] Е. М. Ильгенфриц, С. А. Пастон, Г. Ю. Пирнер, Е. В. Прохватилов, В. А. Франке, ТМФ, 148:1 (2006), 89-101, arXiv: hep-th/0610020.

[8] P. Fonseca, A. Zamolodchikov, J. Statist. Phys., 110:3-6 (2003), 527-590, arXiv: hep-th/ 0112167.

[9] P. Fonseca, A. Zamolodchikov, Ising spectroscopy I: Mesons at $T<T_{c}$, arXiv: hep-th/ 0612304.

[10] S.S. Chabysheva, J.R. Hiller, A dynamical model for longitudinal wave functions in light-front holographic QCD, arXiv: 1207.7128.

[11] S. J. Brodsky, G. F. de Téramond, Phys. Rev. Lett., 96:20 (2006), 201601, 4 pp., arXiv: hep-ph/0602252.

[12] S. J. Brodsky, G. F. de Téramond, AdS/CFT and light-front QCD, arXiv: 0802.0514..

[13] J. Mondejar, A. Pineda, Phys. Rev. D, 79:8 (2009), 085011, 30 pp., arXiv: 0901.3113.

[14] M. Shifman, "Quark-hadron duality", At the Frontier of Particle Physics -Handbook of QCD: Boris Ioffe Festschrift, 3, ed. M. Shifman, World Sci., Singapore, 2001, 1577-1670, arXiv: hep-ph/0009131.

[15] H. Lewy, Manuscr. Math., 26:4 (1979), 411-421.

[16] H. Lewy, Indiana Univ. Math. J., 6:1 (1957), 91-107.

[17] A. J. Hanson, R. D. Peccei, M. K. Prasad, Nucl. Phys. B, 121:3 (1977), 477-504.

[18] S. Huang, J. W. Negele, J. Polonyi, Nucl. Phys. B, 307:4 (1988), 669-704.

[19] R. L. Jaffe, P. F. Mende, Nucl. Phys. B, 369:1 (1992), 189-218. 
[20] W. Krauth, M. Staudacher, Phys. Lett. B, 388:4 (1996), 808-812, arXiv: hep-th/9608122.

[21] S. Hildebrandt, Manuscr. Math., 24:1 (1978), 45-79.

[22] S. Hildebrandt, Ark. Mat., 17:1-2 (1979), 29-38.

[23] S. Hildebrandt, V. Višnjić-Triantafillou, Math. Z., 168:3 (1979), 223-240.

[24] J. Bruning, Manuscr. Math., 39:1 (1982), 125-146.

[25] I. Ziyatdinov, Internat. J. Modern Phys. A, 25:20 (2010), 3899-3910, arXiv: 1003.4304.

[26] V.A. Fateev, S. L. Lukyanov, A. B. Zamolodchikov, J. Phys. A, 42:30 (2009), 304012, 23 pp., arXiv: 0905.2280.

[27] М. Ю. Малышев, Е. В. Прохватилов, ТМФ, 169:2 (2011), 272-284.

[28] М. Абрамовиц, И. Стиган (ред.), Справочник по специальным функциям с формулами, графиками и математическими таблищами, Наука, М., 1979. 